\title{
Serum antibody response in patients with philadelphia- chromosome positive or negative myeloproliferative neoplasms following vaccination with SARS-CoV-2 spike protein messenger RNA (mRNA) vaccines
}

Kathryn E. Kozak' ${ }^{1}$ Linda Ouyang ${ }^{1}$, Andriy Derkach ${ }^{2}$, Alexandra Sherman' ${ }^{1}$ Susan J. McCall' ${ }^{1}$ Christopher Famulare ${ }^{3}$, Jordan Chervin (10 ${ }^{3}$, Ryan J. Daley ${ }^{1}$, Sejal Morjaria ${ }^{4}$, Michael J. Mauro $\mathbb{1}^{1 凶}$ and Raajit K. Rampal $\mathbb{B}^{1,3 凶}$

(C) The Author(s), under exclusive licence to Springer Nature Limited 2021

Leukemia (2021) 35:3578-3580; https://doi.org/10.1038/s41375-021-01457-1

\section{TO THE EDITOR:}

Vaccination against severe acute respiratory syndrome coronavirus 2 (SARS-CoV-2), particularly spike protein messenger RNA (mRNA) vaccines, to date have yielded a high degree of efficacy against symptomatic infection and severe Covid-19 [1, 2]. However, outcomes of patients with active hematologic malignancies are not well described, and remain a significant concern given data demonstrating poorer outcomes in sub-groups of these patients who become infected [3]; for example, a recent study of patients with chronic lymphocytic leukemia (CLL) demonstrated reduced antibody seroprevalence after vaccination in patients on active therapy with Burton Tyrosine Kinase (BTK) inhibitors [4]. These observations prompted us to evaluate post-vaccination antibody response in patients with Philadelphia-chromosome positive and negative Myeloproliferative Neoplasms (MPNs), including untreated patients and patients on active therapy.

We evaluated antibody response in 74 consecutive patients with MPNs subsequent to two doses of the mRNA BNT162b2 (Pfizer) or mRNA-1273 (Moderna) vaccines, as per the U.S. Food and Drug Administration Emergency Use Authorization (EUA). We included patients with chronic myeloid leukemia (CML), essential thrombocythemia (ET), polycythemia vera (PV), myelofibrosis (MF), or blastphase MPN (MPN-BP). Serology testing was performed as part of routine clinical practice using the AdviseDx SARS-CoV-2 lgG II reagent assay (Abbott Diagnostics). A result was considered positive with $\geq 50 \mathrm{AU} / \mathrm{mL}$. The study was approved by the Institutional Review Board of Memorial Sloan Kettering Cancer Center.

Data were collected from a period spanning from April 2021 to September 2021, from 74 patients in total. The cohort consisted of 22 patients with CML, 14 patients with ET, 14 patients with PV, 22 patients with MF, and 2 patients with MPN-BP (both transformed from prior post-ET Myelofibrosis). The median age of included subjects was 68.2 years. Eighteen $C M L$ patients were receiving active treatment with $A B L$ kinase inhibitors, including dasatinib
(7/22), imatinib (5/22), bosutinib (2/22), ponatinib (1/22), nilotinib $(2 / 22)$ and combination nilotinib and ruxolitinib (on clinical trial, $1 / 22$ ). Four CML patients were being observed on treatment-free remission (4/22). Amongst ET patients, 5/14 were not receiving active cytoreductive therapy (once daily aspirin only); $5 / 14$ were receiving hydroxyurea, and 4/14 were receiving pegylated interferon. Therapeutic phlebotomy and aspirin only were utilized in 6/14 PV patients, hydroxyurea in 6/14, pegylated interferon in $1 / 14$ and ruxolitinib in $1 / 14$. Amongst MF patients, $11 / 22$ were on no active treatment, $8 / 22$ were being treated with the JAK1/2 inhibitor ruxolitinib, 2/22 were receiving treatment with an investigational regimen (ruxolitinib combined with a BET inhibitor or a single agent TGF- $\beta$ inhibitor), and $1 / 22$ patients was receiving hydroxyurea. No patients studied had a clinical history of Covid-19 infection. All patients in the cohort studied received either the BNT162b2 or mRNA-1273 vaccines. The median time from last dose of vaccination to measurement of serum antibody response was 100 days.

Antibody seropositivity was observed in all disease categories with no statistical differences between disease types (Fig. 1A). Amongst patients with $C M L$, no differences were noted in spike protein antibody levels between $\mathrm{ABL}$ kinase inhibitors utilized (Fig. 1B). In the ET cohort, no statistical differences were noted between treatment subgroups. Notably, however, spike protein antibody levels trended higher in the pegylated interferon treated patients (mean $4151.15 \mathrm{AU} / \mathrm{mL}$ ) than in patients treated with hydroxyurea (mean 2368.62) or patients on aspirin only (2447.52; Fig. 1C). No differences were noted in spike protein antibody levels between cytoreduced and non-cytoreduced PV patients (Fig. 1D). In the MF cohort, nominally lower levels of spike protein antibody were noted when comparing patients on ruxolitinib (mean 603.1) versus patients on observation (mean 6353.29; Fig. 1E), although this difference also did not meet statistical significance.

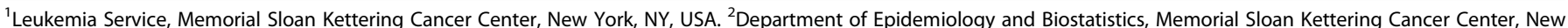

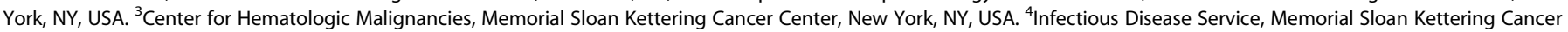
Center, New York, NY, USA. ${ }^{凶}$ email: maurom@mskcc.org; rampalr@mskcc.org
} 
A

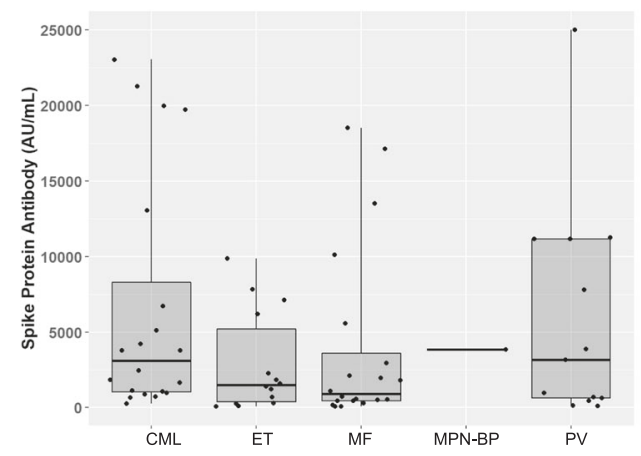

C

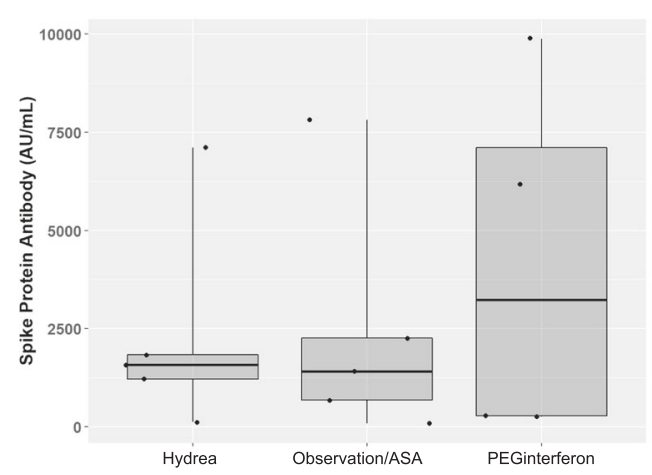

$E$

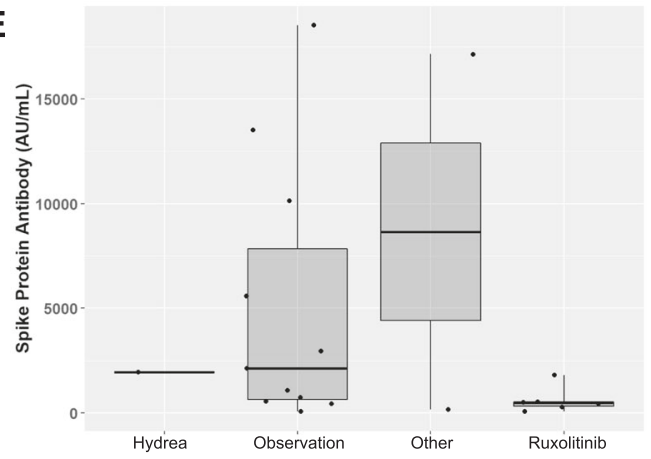

G

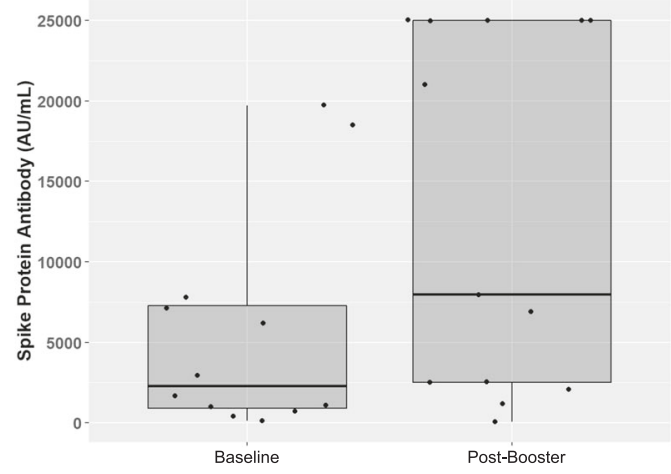

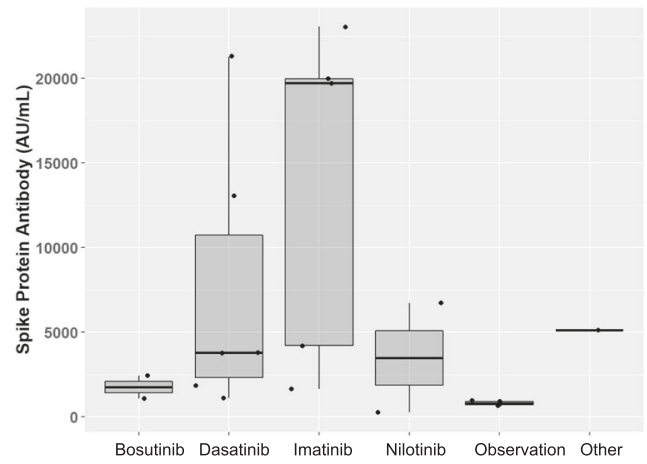

D

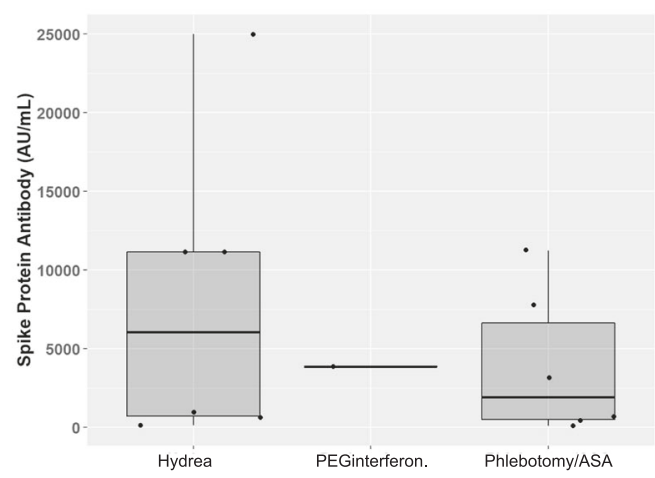

$\mathbf{F}$

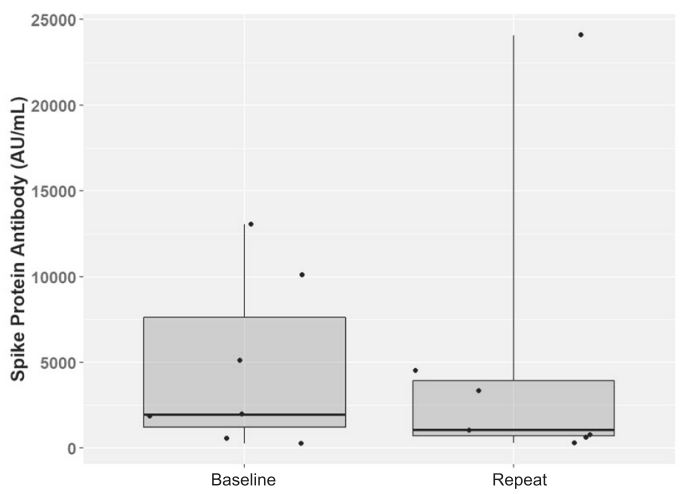

Fig. 1 Spike protein antibody level. A Spike protein antibody levels across all disease types studied. Spike protein antibody levels by cytoreductive therapy in CML patients (B) ET patients (C) PV patients (D) and MF patients (E). Serial spike protein antibody levels (F). Effect of booster vaccination $(\mathbf{G})$.

In seven patients, serial assessment of spike protein antibody levels over time was available. In $6 / 7$ cases we observed a reduction in antibody level from the time of initial assessment, with mean level of decrease of $67 \%$ (Fig. 1F) at a medium of 6 months from completion of their initial vaccination series.
We also assessed the response to administration of a $3^{\text {rd }}$ dose of the mRNA vaccines (booster dose) in 13 patients, who received a booster dose at a medium of 6.8 months from completion of their initial vaccination series. A median level of increase in the spike protein antibody level of $200 \%$ was observed in this cohort (Fig. 1G). 
Of all patients studied, three patients were identified who lacked measurable antibody response to vaccination (defined as level less than $50 \mathrm{AU} / \mathrm{mL}$ ), one with $\mathrm{CML}$ on ponatinib, one with $\mathrm{PV}$ on ruxolitinib, and one with MPN-BP on ruxolitinib.

To date, limited data have been published with regards to outcomes of MPN patients diagnosed with Covid-19. One study demonstrated mortality of $28.6 \%$ in MPN patients diagnosed with Covid-19 at a median time of 9.5 days after diagnosis [5]. Mortality was highest in patients with MF (48\%) and linked to discontinuation of ruxolitinib. Longer-term follow-up of MPN patients with Covid-19 infection has demonstrated that $40 \%$ of patients experience fatal and non-fatal events in the 6 months after initial infection [6]. Thus, preventing Covid-19 infection, particularly severe infection requiring hospitalization, is of great importance in patients with MPNs.

A prior study has examined seropositivity in patients with MPNs and MDS inoculated with ChAdOx1 nCoV-19 vaccine (AztraZenecaOxford) or a single dose of the BNT162b2 vaccine, which demonstrated that seroconversion in MPN and MDS patients was significantly lower when compared to health care worker controls (58\% versus $97 \%$ ) [7]. By contrast, our study focused on patients who received full (two doses) mRNA based vaccination as outlined by the EUA. The overwhelming majority of patients studied (71/74) developed measurable spike protein antibodies. While two patients who did not convert to seropositive were both treated with ruxolitinib, in both cases, there are potential confounding variables; one patient had previously received a PI3K delta inhibitor in addition to ruxolitinib, and the other patient had repeated systemic steroid exposure for treatment of sinusitis. While a trend toward lower spike-protein antibody levels was noted in MF patients on ruxolitinib in our cohort, overall seven of nine patients on ruxolitinib did develop detectable spike protein antibodies.

Limitations of our study include a relatively limited patient cohort, restricting our ability to determine potential distinct differences between MPN-directed treatments within each disease subgroup in regard to vaccination response.

We conclude that two doses of SARS-CoV-2 spike protein messenger RNA (mRNA) vaccine results in a high rate of seroconversion in MPN patients, including those treated with cytoreductive therapies. Additional analyses of patients in our cohort revealed that while antibody levels appear to decrease over time, a booster ( $3 \mathrm{rd}$ ) dose of these vaccines was able to increase antibody levels in the majority. The degree of protection against Covid-19 afforded by the noted levels of antibody response remains unclear in this and other populations. Further studies of larger numbers of patients over an extended follow-up period are needed to determine the clinical impacts of seroconversion.

\section{REFERENCES}

1. Polack FP, Thomas SJ, Kitchin N, Absalon J, Gurtman A, Lockhart S, et al. Safety and efficacy of the BNT162b2 mRNA Covid-19 vaccine. N Engl J Med. 2020;383:2603-15.

2. Baden LR, El Sahly HM, Essink B, Kotloff K, Frey S, Novak R, et al. Efficacy and Safety of the mRNA-1273 SARS-CoV-2 Vaccine. N Engl J Med. 2021;384:403-16.

3. Mato AR, Roeker LE, Lamanna N, Allan JN, Leslie L, Pagel JM, et al. Outcomes of COVID-19 in patients with CLL: a multicenter international experience. Blood. 2020;136:1134-43.

4. Roeker LE, Knorr DA, Thompson MC, Nivar M, Lebowitz S, Peters N, et al. COVID-19 vaccine efficacy in patients with chronic lymphocytic leukemia. Leukemia. 2021;35:2703-5.

5. Barbui T, Vannucchi AM, Alvarez-Larran A, lurlo A, Masciulli A, Carobbio A, et al. High mortality rate in COVID-19 patients with myeloproliferative neoplasms after abrupt withdrawal of ruxolitinib. Leukemia. 2021;35:485-93.

6. Barbui T, lurlo A, Masciulli A, Carobbio A, Ghirardi A, Rossi G, et al. Long-term follow-up of recovered MPN patients with COVID-19. Blood Cancer J. 2021;11:115.

7. Chowdhury $\mathrm{O}$, Bruguier H, Mallett $\mathrm{G}$, Sousos N, Crozier K, Allman C, et al. Impaired antibody response to COVID-19 vaccination in patients with chronic myeloid neoplasms. Br J Haematol. 2021;194:1010-5.

\section{ACKNOWLEDGEMENTS}

This research was supported in part by the $\mathrm{NIH} / \mathrm{NCl}$ Cancer Center Support Grant P30 CA008748.

\section{AUTHOR CONTRIBUTIONS}

Data collection and analysis was carried out by all authors. Statistical analysis was carried by AD. Manuscript was written by RKR, MJM, and RJD.

\section{COMPETING INTERESTS}

RKR has received consulting fees from: Constellation, Novartis, Kartos, Incyte, Celgene/BMS, Promedior, CTI, Blueprint, Stemline, Galecto, Pharmaessentia, Sierra Oncology, Abbvie and research funding from Incyte, Constellation, Stemline. MJM has received consulting/advising fees from Novartis, Bristol Myers Squibb, Takeda, and Pfizer and research funding (institutional) from Novartis, Bristol Myers Squibb, and Sun Pharma/SPARC.

\section{ADDITIONAL INFORMATION}

Correspondence and requests for materials should be addressed to Michael J. Mauro or Raajit K. Rampal.

Reprints and permission information is available at http://www.nature.com/ reprints

Publisher's note Springer Nature remains neutral with regard to jurisdictional claims in published maps and institutional affiliations. 